1 Hacettepe Journal of Mathematics and Statistics

holume 45 (1) (2016), 69-76

\title{
Comparison of near sets by means of a chain of features
}

\author{
A. Fatih Özcan* and Nurettin Bă̆ırmaz ${ }^{\dagger \ddagger}$
}

\begin{abstract}
If the number of features of objects in a perceptual system, is large, then the objects can be known better and comparable. In this paper basically, we form a chain of feature sets that describe objects and then by means of this chain of feature sets, we investigate the nearness of sets and near sets in a perceptual system.
\end{abstract}

Keywords: Near set, Feature chain, Indiscernibility, Nearness.

2000 AMS Classification: 03E75, 03E99, 03E02

Received: 03.01.2013 Accepted: 15.01.2015 Doi: 10.15672/HJMS.20164512480

\section{Introduction}

Near sets were introduced by J.F. Peters [11], which are indeed a form of generalization of rough sets proposed by Z. Pawlak [6]. The algebraic properties of near sets are described in [9]. Recent work has considered near soft sets [20], soft nearness approximation spaces [4], near groups [3], isometries in proximity spaces [18], and applications of near sets $[17,19]$. The fundamental idea of near set theory is object description and classification according to perceptual knowledge. It is supposed that perceptual knowledge about objects is always given with respect to probe functions, i.e., real-valued functions which represent features of a physical object. Some well known examples of probe functions are the colour, size or weight of an object $[1,2,9-16,21]$.

*Inonu University, Science and Art Faculty, Departmant of Mathematics, Maltya, Turkey, Email: abdullah.ozcan@inonu.edu.tr

${ }^{\dagger}$ Mardin Artuklu University, Vocational Higher Schools of Mardin, Mardin, Turkey, Email: nurettinbagirmaz@artuklu.edu.tr

${ }^{\ddagger}$ Corresponding Author. 


\section{Preliminiaries}

In this section, we present the basic definitions of near set theory [9,11]. More detailed explanations related to near sets and rough sets can be found in [1,2,9-16,21] and [5-8], respectively.

2.1. Definition. [9] (Perceptual Object) A perceptual object is something perceivable that has its origin in the physical world.

2.2. Definition. [9] (Probe Function) A probe function is a real-valued function representing a feature of a perceptual object.Simple examples of probe functions are the colour, size or weight of an object.

2.3. Definition. [9] (Perceptual System) A perceptual system $\langle O, F\rangle$ consists of a non-empty set $O$ of sample perceptual objects and a non-empty set $F$ of real-valued functions $\phi \in F$ such that $\phi: O \rightarrow \mathbb{R}$.

2.4. Definition. [9] (Object Description) Let $\langle O, F\rangle$ be a perceptual system, and let $B \subseteq F$ be a set of probe functions. Then, the description of a perceptual object $x \in O$ is a feature vector given by

$$
\phi_{B}(x)=\left(\phi_{1}(x), \phi_{2}(x), \ldots, \phi_{i}(x), \ldots, \phi_{l}(x)\right)
$$

where $l$ is the length of the vector $\phi_{B}$, and each $\phi_{i}(x)$ in $\phi_{B}(x)$ is a probe function value that is part of the description of the object $x \in O$.

2.5. Definition. $[2,6]$ (Indiscernibility relation) Let $\langle O, F\rangle$ be a perceptual system. For every $B \subseteq F$ the indiscernibility relation $\sim_{B}$ is defined as follows:

$$
\sim_{B}=\left\{(x, y) \in O \times O \mid \forall \phi_{i} \in B, \phi_{i}(x)=\phi_{i}(y)\right\} .
$$

If $B=\{\phi\}$ for some $\phi \in F$, instead of $\sim_{\{\phi\}}$ we write $\sim_{\phi}$.

The indiscernibility relation $\sim_{B}$ is an equivalence relation on object descriptions.

2.6. Lemma. [9] Let $\langle O, F\rangle$ be a perceptual system. For every $B \subseteq F$,

$$
\sim_{B}=\bigcap_{\phi \in B} \sim_{\phi}
$$

2.7. Definition. (Equivalence Class) Let $\langle O, F\rangle$ be a perceptual system and let $x \in O$ . For a set $B \subseteq F$ an equivalence class is defined as $x / \sim_{B}=\left\{y \in O \mid y \sim_{B} x\right\}$.

2.8. Definition. (Quotient Set)Let $\langle O, F\rangle$ be a perceptual system. For a set $B \subseteq F$ a quotient set is defined as

$$
O / \sim_{B}=\left\{x / \sim_{B} \mid x \in O\right\} .
$$

2.9. Definition. [9] Let $\langle O, F\rangle$ be a perceptual system. Then

$$
\prod(O, F):=\bigcup_{B \subseteq F} O / \sim_{B}
$$

i.e., $\prod(O, F)$ is the family of equivalence classes of all indiscernibility relations determined by a perceptual information system $\langle O, F\rangle$.

2.10. Definition. [9] (Nearness relation). Let $\langle O, F\rangle$ be a perceptual system and let $X, Y \subseteq O$. A set $X$ is near to a set $Y$ within the perceptual system $\langle O, F\rangle\left(X \bowtie_{F} Y\right)$ iff there are $F_{1}, F_{2} \subseteq F$ and $f \in F$ and there are $A \in O / \sim_{F_{1}}, B \in O / \sim_{F_{2}}, C \in O / \sim f$ such that $A \subseteq X, B \subseteq Y$ ve $A, B \subseteq C$.If a perceptual system is understood, then we say briefly that a set $X$ is near to a set $Y$. 
2.11. Definition. [9] (Perceptual near sets) Let $\langle O, F\rangle$ be a perceptual system and let $X \subseteq O$. A set $X$ is a perceptual near set iff there is $Y \subseteq O$ such that $X \bowtie_{F} Y$. The family of near sets of a perceptual system $\langle O, F\rangle$ is denoted by $\operatorname{Near}_{F}(O)$.

2.12. Example. Let $\langle O, F\rangle$ be a perceptual system such that $O=\left\{x_{1}, x_{2}, \ldots, x_{6}\right\}$, $F=\left\{\phi_{1}, \phi_{2}\right\}, \phi_{1}\left(x_{1}\right)=\phi_{1}\left(x_{2}\right)=\phi_{1}\left(x_{3}\right), \phi_{1}\left(x_{4}\right)=\phi_{1}\left(x_{5}\right)=\phi_{1}\left(x_{6}\right), \phi_{1}\left(x_{1}\right) \neq \phi_{1}\left(x_{4}\right)$ and $\phi_{2}\left(x_{1}\right)=\phi_{2}\left(x_{2}\right), \phi_{2}\left(x_{3}\right)=\phi_{2}\left(x_{4}\right), \phi_{2}\left(x_{5}\right)=\phi_{2}\left(x_{6}\right), \phi_{2}\left(x_{1}\right) \neq \phi_{2}\left(x_{4}\right) \neq \phi_{2}\left(x_{5}\right)$.

Thus $O / \sim_{\phi_{1}}=\left\{\left\{x_{1}, x_{2}, x_{3}\right\},\left\{x_{4}, x_{5}, x_{6}\right\}\right\}, O / \sim_{\phi_{2}}=\left\{\left\{x_{1}, x_{2}\right\},\left\{x_{3}, x_{4}\right\},\left\{x_{5}, x_{6}\right\}\right\}$, $O / \sim_{\left\{\phi_{1}, \phi_{2}\right\}}=\left\{\left\{x_{1}, x_{2}\right\},\left\{x_{3}\right\},\left\{x_{4}\right\},\left\{x_{5}, x_{6}\right\}\right\}$.

Let $X=\left\{x_{1}, x_{2}, x_{3}, x_{5}\right\}, Y=\left\{x_{2}, x_{4}, x_{5}, x_{6}\right\}$.Thus there are $A=\left\{x_{4}\right\} \in O / \sim_{\left\{\phi_{1}, \phi_{2}\right\}}$, $B=\left\{x_{5}, x_{6}\right\} \in O / \sim_{\phi_{2}} C=(A \cup B) \in O / \sim_{\phi_{1}}$ such that $A \subseteq X, B \subseteq Y$. Therefore $X \bowtie_{F} Y$.

2.13. Proposition. [9] Let $\langle O, F\rangle$ be a perceptual system, $B \subseteq F$ and $x / \sim_{B} \in O / \sim_{B}$, where $\left|x / \sim_{B}\right| \geq 2$. All elements belonging to a class $x / \sim_{B}$ are near each other.

2.14. Proposition. [9] Let $\langle O, F\rangle$ be a perceptual system. For any $B \subseteq F$, every equivalence class of an indiscernibility relation $\sim_{B}$ is a near set.

\section{Some New Properties of Near Sets}

In this section, we give some new propositions which are related to some propositions in [9].

3.1. Proposition. [9] Let $\langle O, F\rangle$ be a perceptual system. For every $X \subseteq O$, the following conditions are equivalent:

(1) $X \in \operatorname{Near}_{F}(O)$,

(2) there is $A \in \prod(O, F)$ such that $A \subseteq X$,

(3) there is $A \in O / \sim_{F}$ such that $A \subseteq X$.

3.2. Proposition. Let $\langle O, F\rangle$ be a perceptual system and $X, Y \subseteq O$. Then

$$
X \bowtie_{F} Y \Rightarrow X, Y \in \operatorname{Near}_{F}(O) \text {. }
$$

Proof. Let $X \bowtie_{F} Y$. From Definition 2.11, there are $A, B \in \prod(O, F)$ such that $A \subseteq$ $X, B \subseteq Y$.Thus, from Proposition 3.1, $X, Y \in \operatorname{Near}_{F}(O)$.

3.3. Remark. From Proposition 3.2, two near sets may not be near to each other. We can see this in the following example.

3.4. Example. Let $\langle O, F\rangle$ be a perceptual system such that $O=\left\{x_{1}, x_{2}, \ldots, x_{6}\right\}$,for simplicity $F=(\phi)$ and $\phi\left(x_{2}\right)=\phi\left(x_{3}\right), \phi\left(x_{4}\right)=\phi\left(x_{5}\right)=\phi\left(x_{6}\right), \phi\left(x_{1}\right) \neq \phi\left(x_{2}\right) \neq$ $\phi\left(x_{4}\right)$. Thus $O / \sim_{\phi}=\left\{\left\{x_{1}\right\},\left\{x_{2}, x_{3}\right\},\left\{x_{4}, x_{5}, x_{6}\right\}\right\}$. Let $X=\left\{x_{1}, x_{2}\right\}, Y=\left\{x_{2}, x_{3}, x_{6}\right\}$. There are $A=\left\{x_{1}\right\} \in O / \sim \phi, B=\left\{x_{2}, x_{3}\right\} \in O / \sim \phi$ such that $A \subseteq X, B \subseteq Y$, so $X, Y \in$ $\operatorname{Near}_{F}(O)$. But there is no $C \in O / \sim \phi$ such that $A, B \subseteq C$. Therefore $X$ and $Y$ are not near to each other.

3.5. Proposition. [9] Let $\langle O, F\rangle$ be a perceptual system and $X, Y \subseteq O$. Then

$$
X, Y \in \operatorname{Near}_{F}(O) \Rightarrow X \cup Y \in \operatorname{Near}_{F}(O),
$$

i.e., the family of near sets of a perceptual system $\langle O, F\rangle$ is closed for the union of sets.

3.6. Proposition. Let $\langle O, F\rangle$ be a perceptual system and $X, Y \subseteq O$. Then

$$
X \bowtie_{F} Y \Rightarrow X \cup Y \in \operatorname{Near}_{F}(O) .
$$

Proof. It is clear from Proposition 3.2 and Proposition 3.5 . 
3.7. Proposition. [9] Let $\langle O, F\rangle$ be a. Then

$$
X \in \prod(O, F) \Rightarrow X \bowtie_{F} X,
$$

i.e., the relation $\bowtie_{F}$ is reflexive within the family $\prod(O, F)$.

3.8. Proposition. Let $\langle O, F\rangle$ be a perceptual system. Then

$X \bowtie_{F} X \Leftrightarrow$ there is $A \in \prod(O, F)$ such that $A \subseteq X$.

That is, a set $X \subseteq O$ to be near to itself need not be a equivalence class or need not be a union of equivalence classes. But at least it has to contain an equivalence class.

Proof. It is clear.

3.9. Proposition. [9] Let $\langle O, F\rangle$ be a perceptual system. For any $X, Y \subseteq O$, if there is $A \in \prod(O, F)$ such that $A \subseteq X \cap Y$, then $X \bowtie_{F} Y$.

3.10. Proposition. Let $\langle O, F\rangle$ be a perceptual system and let $X, Y \subseteq O$ and $F$ is a singleton set. Then

$X \bowtie_{F} Y \Leftrightarrow$ there is $A \in \prod(O, F)$ such that $A \subseteq X \cap Y$.

Proof. It is enough to prove the implication $(\Rightarrow)$. From Definition 2.10, there are $A \in$ $O / \sim_{F}, B \in O / \sim_{F}, C \in O / \sim_{F}$ such that $A \subseteq X, B \subseteq Y$ and $A, B \subseteq C$. Since $F$ is a singleton set and $A, B \subseteq C$, then $A=B=C$. Therefore $A \subseteq X \cap Y$.

3.11. Proposition. [9] Let $\langle O, F\rangle$ be a perceptual system and let $X, Y, Z \subseteq O$. Then the following conditions hold:

(1) $X \bowtie_{F} Y \& Y \subseteq Z \Rightarrow X \bowtie_{F} Z$,

(2) $X \subseteq Y \& X \bowtie_{F} Z \Rightarrow Y \bowtie_{F} Z$.

3.12. Proposition. Let $\langle O, F\rangle$ be a perceptual system and $A_{1}, A_{2}, B_{1}, B_{2} \subseteq O$. Then the following conditions hold:

(1) $A_{1} \bowtie_{F} A_{2} \& B_{1} \bowtie_{F} B_{2} \Rightarrow\left(A_{1} \cup B_{1}\right) \bowtie_{F}\left(A_{2} \cup B_{2}\right)$ or $\left(A_{1} \cup B_{2}\right) \bowtie_{F}\left(A_{2} \cup B_{1}\right)$,

(2) $\left(A_{1} \cap A_{2}\right) \bowtie_{F}\left(B_{1} \cap B_{2}\right) \Rightarrow A_{1} \bowtie_{F} B_{1}$ or $A_{1} \bowtie_{F} B_{2}$ or $A_{2} \bowtie_{F} B_{1}$ or $A_{2} \bowtie_{F} B_{2}$.

Proof. Let $\langle O, F\rangle$ be a perceptual system and let $A_{1}, A_{2}, B_{1}, B_{2} \subseteq O$.

Case (1). Let $A_{1} \bowtie_{F} A_{2}$ and $B_{1} \bowtie_{F} B_{2}$. So $A_{1} \bowtie_{F} A_{2}, A_{2} \subseteq A_{2} \cup B_{2}$ and $B_{1} \bowtie_{F} B_{2}, B_{2} \subseteq\left(A_{2} \cup B_{2}\right)$ then from Proposition 3.11 (1) $A_{1} \bowtie_{F}\left(A_{2} \cup B_{2}\right)$ and $B_{1} \bowtie_{F}$ $\left(A_{2} \cup B_{2}\right)$. Since $A_{1} \bowtie_{F}\left(A_{2} \cup B_{2}\right)$ and $B_{1} \bowtie_{F}\left(A_{2} \cup B_{2}\right),\left(A_{1} \cup B_{1}\right) \bowtie_{F}\left(A_{2} \cup B_{2}\right)$. Similarly it can be shown that $\left(A_{1} \cup B_{2}\right) \bowtie_{F}\left(A_{2} \cup B_{1}\right)$.

Case (2). Let $\left(A_{1} \cap A_{2}\right) \bowtie_{F}\left(B_{1} \cap B_{2}\right)$. Since $\left(A_{1} \cap A_{2}\right) \subseteq A_{1}$ and from Proposition 3.11 (2) $A_{1} \bowtie_{F}\left(B_{1} \cap B_{2}\right)$. Since $A_{1} \bowtie_{F}\left(B_{1} \cap B_{2}\right)$ and from Proposition 3.11 (1), then $A_{1} \bowtie_{F} B_{1}$. Similarly it can be shown that $A_{2} \bowtie_{F} B_{1}$ or $A_{2} \bowtie_{F} B_{1}$ or $A_{2} \bowtie_{F} B_{2}$.

The fact that the reverse of the implication reversed in Proposition 3.12 (1) does not hold is shown by example. Similarly it can be shown that the Proposition 3.12 (2) does not hold always.

3.13. Example. Let $\langle O, F\rangle$ be a perceptual system such that $O=\left\{x_{1}, x_{2}, \ldots, x_{8}\right\}$, so $O / \sim_{F}=\left\{\left\{x_{1}, x_{2}, x_{3}\right\},\left\{x_{4}, x_{5}\right\},\left\{x_{6}, x_{7}, x_{8}\right\}\right\}$.Let $A_{1}=\left\{x_{2}, x_{3}, x_{4}\right\}, A_{2}=\left\{x_{1}, x_{2}, x_{3}, x_{5}\right\}$, $B_{1}=\left\{x_{1}, x_{3}, x_{4}, x_{7}\right\}, B_{2}=\left\{x_{2}, x_{4}, x_{6}, x_{8}\right\}$,so $A_{1} \cup B_{1}=\left\{x_{1}, x_{2}, x_{3}, x_{4}, x_{7}\right\}$ and $A_{2} \cup$ $B_{2}=\left\{x_{1}, x_{2}, x_{3}, x_{4}, x_{5}, x_{6}, x_{8}\right\}$. Since $\left\{x_{1}, x_{2}, x_{3}\right\} \in O / \sim_{F}$ and $\left\{x_{1}, x_{2}, x_{3}\right\} \subseteq A_{1} \cup B_{1}, A_{2} \cup$ $B_{2} A_{1} \cup B_{1} \bowtie_{F} A_{2} \cup B_{2}$. But there is no $X / \sim_{F} \in O / \sim_{F}, Y / \sim_{F} \in O / \sim_{F}, Z / \sim_{F} \in$ $O / \sim_{F}$ such that $X_{/ \sim_{F}} \subseteq A_{1}, Y_{/ \sim_{F}} \subseteq A_{2}$ and $X, Y \subseteq Z$. Therefore, from Definition 2.10, $A_{1}$ and $A_{2}$ are not near to each other. For same reason, $B_{1}$ and $B_{2}$ are not near to each other. 


\section{Chain of Features, Nearness and Near Sets}

In this section basically, a nested chain of probe functions (features) is formed and corresponding indiscernibility relation, nearness relation and near sets in $\langle O, F\rangle$ perceptual system are investigated.

4.1. Definition. Let $\langle O, F\rangle$ be a perceptual system. Then

$\prod\left(O, \sim_{F}\right):=\left\{\sim_{B} \mid B \subseteq F\right\}$,

i.e. $\prod\left(O, \sim_{F}\right)$ is the family of indiscernibility relations of all probe functions determined by a perceptual information system $\langle O, F\rangle$.

4.2. Lemma. Let $\langle O, F\rangle$ be a perceptual system, $\Pi(O, F)$ is the family of equivalence classes of all indiscernibility relations and $\prod\left(O, \sim_{F}\right)$ is the family of indiscernibility relations of all probe functions. Then for all $B \subseteq F$, the function

$$
\begin{aligned}
f: \prod\left(O, \sim_{F}\right) & \rightarrow \prod(O, F) \\
\sim_{B} & \mapsto O / \sim_{B}
\end{aligned}
$$

is one-to-one and onto.

4.3. Proposition. Let $\langle O, F\rangle$ be a perceptual system and $F=B_{n}=\left\{\phi_{1}, \phi_{2}, \ldots, \phi_{n}\right\}$. Then for all $B_{i} \subseteq F, 1 \leq j, i \leq n$,

$$
B_{j} \subseteq B_{i} \Leftrightarrow \sim_{B_{i}} \subseteq \sim_{B_{j}} \text {. }
$$

Proof. Let $B_{i} \subseteq F \quad, B_{j} \subseteq B_{i}, 1 \leq j, i \leq n$. Since $\bigcap_{\phi \in B_{j}} \sim_{\phi} \subseteq \bigcap_{\phi \in B_{i}} \sim_{\phi}$ and, from Lemma 2.6, $\sim_{B_{i}} \subseteq \sim_{B_{j}}$.

4.4. Corollary. Let $\langle O, F\rangle$ be a perceptual system and $F=B_{n}=\left\{\phi_{1}, \phi_{2}, \ldots, \phi_{n}\right\}$. Then for all $B_{i} \subseteq F, B_{j} \subseteq B_{i}, 1 \leq j, i \leq n$,

$$
\sim_{B_{i}} \subseteq \sim_{B_{j}} \Leftrightarrow \underset{\phi \in B_{i}}{\bigcap} \sim_{\phi} \subseteq \underset{\phi \in B j}{\bigcap} \sim_{\phi}
$$

4.5. Proposition. Let $\langle O, F\rangle$ be a perceptual system, $F=B_{n}=\left\{\phi_{1}, \phi_{2}, \ldots, \phi_{n}\right\}$ and $B_{i} \subseteq F \quad, B_{j} \subseteq B_{i}, 1 \leq j, i \leq n$. Then

that $A \subseteq C$.

$\sim_{B_{i}} \subseteq \sim_{B_{j}} \Rightarrow$ For all $A \in O / \sim_{B_{i}}$ there is a unique $C \in O / \sim_{B_{j}}$ such

Proof. Let $\sim_{B_{i}} \subseteq \sim_{B_{j}}, x \in O, A=x / \sim_{B_{i}}$ and $C=x / \sim_{B_{j}}$. Since $\sim_{B_{i}} \subseteq \sim_{B_{j}}$, then $x / \sim_{B_{i}} \subseteq$ $x / \sim_{B_{j}}$.

4.6. Proposition. Let $\langle O, F\rangle$ be a perceptual system, $X \subseteq O, F=B_{n}=\left\{\phi_{1}, \phi_{2}, \ldots, \phi_{n}\right\}$ and $B_{i} \subseteq F \quad, B_{j} \subseteq B_{i}, 1 \leq j, i \leq n$. Then the following conditions hold:

$$
\begin{aligned}
& \text { (1) } \prod\left(O, \sim_{B_{j}}\right) \subseteq \prod\left(O, \sim_{B_{i}}\right), \\
& \text { (2) } \prod\left(O, B_{j}\right) \subseteq \prod\left(O, B_{i}\right) .
\end{aligned}
$$

Proof. Let $\langle O, F\rangle$ be a perceptual system , $X \subseteq O, F=B_{n}=\left\{\phi_{1}, \phi_{2}, \ldots, \phi_{n}\right\}$ and $B_{i} \subseteq F \quad, B_{j} \subseteq B_{i}, 1 \leq j, i \leq n$.

(1) Since $B \subseteq B_{j}$ then $B \subseteq B_{i}$. Thus from Definition $4.1 \prod\left(O, \sim_{B_{j}}\right) \subseteq \prod\left(O, \sim_{B_{i}}\right)$.

(2) Since $B_{j} \subseteq B_{i}$, from Definition $2.9 \prod\left(O, B_{j}\right) \subseteq \prod\left(O, B_{i}\right)$.

4.7. Proposition. Let $\langle O, F\rangle$ be a perceptual system,$F=B_{n}=\left\{\phi_{1}, \phi_{2}, \ldots, \phi_{n}\right\}$ and $B_{i} \subseteq F \quad, B_{j} \subseteq B_{i}, 1 \leq j, i \leq n$. Then

$$
\operatorname{Near}_{B_{j}}(O) \subseteq \operatorname{Near}_{B_{i}}(O) .
$$


Proof. Let $X \subseteq O$ and $X \in \operatorname{Near}_{B_{j}}(O)$. Since $X \in \operatorname{Near}_{B_{j}}(O)$ there is $A \in \prod\left(O, \sim_{B_{j}}\right)$ such that $A \subseteq X$. From Proposition 4.6 (1) $A \in \prod\left(O, \sim_{B_{i}}\right)$. Therefore $X \in \operatorname{Near}_{B_{i}}(O)$.

The fact that the reverse of the implication reversed in Proposition 4.7 does not hold. We can see this in the next example.

4.8. Example. Let $\langle O, F\rangle$ be perceptual system in Example 2.12. Thus $O=\left\{x_{1}, x_{2}, \ldots, x_{6}\right\}$, $F=\left\{\phi_{1}, \phi_{2}\right\}$. Recall also that $O / \sim_{\phi_{1}}=\left\{\left\{x_{1}, x_{2}, x_{3}\right\},\left\{x_{4}, x_{5}, x_{6}\right\}\right\}, O / \sim_{\phi_{2}}=\left\{\left\{x_{1}, x_{2}\right\}\right.$ $,\left\{x_{3}, x_{4}\right\},\left\{x_{5}, x_{6}\right\}, O / \sim_{\left\{\phi_{1}, \phi_{2}\right\}}=\left\{\left\{x_{1}, x_{2}\right\},\left\{x_{3}\right\},\left\{x_{4}\right\},\left\{x_{5}, x_{6}\right\}\right\}$. Let $X \subseteq O, B_{1}, B_{2}$ $\subseteq F$ be defined as: $X=\left\{x_{1}, x_{2}, x_{4}\right\}, B_{1}=\left\{\phi_{1}\right\}, B_{2}=\left\{\phi_{1}, \phi_{2}\right\}$. Since $\left\{x_{1}, x_{2}\right\} \in$ $O / \sim_{\left\{\phi_{1}, \phi_{2}\right\}}$ and $\left\{x_{1}, x_{2}\right\} \subseteq X$, then $X \in \operatorname{Near}_{B_{2}}(O)$. But there is no $A \in O / \sim_{\phi_{1}}$ such that $A \subseteq X$, therefore $X \notin \operatorname{Near}_{B_{1}}(O)$.

4.9. Proposition. Let $\langle O, F\rangle$ be a perceptual system , $F=B_{n}=\left\{\phi_{1}, \phi_{2}, \ldots, \phi_{n}\right\}, X, Y \subseteq$ $O$ and $B_{i} \subseteq F \quad, B_{j} \subseteq B_{i}, 1 \leq j, i \leq n$.Then

$$
X \bowtie_{B_{j}} Y \Rightarrow X \bowtie_{B_{i}} Y
$$

Proof. Let $X \bowtie_{B_{j}} Y$. From Definition 2.10 there are $A, B, C \in \prod\left(O, B_{j}\right)$ such that $A \subseteq X, B \subseteq Y$ and $A, B \subseteq C$. Since $A, B, C \in \prod\left(O, B_{j}\right)$, then from Proposition 4.6 (2) $A, B, C \in \prod\left(O, B_{i}\right)$. Again from Definition 2.10, $X \bowtie_{B_{i}} Y$.

4.10. Definition. Let $\langle O, F\rangle$ be a perceptual system, $X, Y \subseteq O, F=B_{n}=\left\{\phi_{1}, \phi_{2}, \ldots, \phi_{n}\right\}$ and $B_{i} \subseteq F$. Then the expression

$X \bowtie_{\sim_{B_{i}}} Y$ means that: A set $X$ is near to a set $Y$ within the perceptual system $\langle O, F\rangle$ only for the $\sim_{B_{i}}$ relation.

4.11. Proposition. Let $\langle O, F\rangle$ be a perceptual system, $X, Y \subseteq O, F=B_{n}=\left\{\phi_{1}, \phi_{2}, \ldots, \phi_{n}\right\}$ and $B_{i} \subseteq F \quad, B_{j} \subseteq B_{i}, 1 \leq j, i \leq n$. Then

$$
X \bowtie_{\sim_{B_{j}}} Y \Rightarrow X \bowtie_{\sim_{B_{i}}} Y .
$$

Proof. Let $X \aleph_{\sim_{B_{j}}} Y$. From Proposition 3.10 and Proposition 3.1, respectively, then $X \cap Y \in \operatorname{Near}_{B_{j}}(O)$. Thus from Proposition 4.7, $X \cap Y \in N e a r_{B_{i}}(O)$. Therefore, from Proposition 3.10, then $X \bowtie_{\sim_{B_{i}}} Y$.

4.12. Example. Let $\langle O, F\rangle$ be perceptual system in the Example 2.12. Recall also that $O / \sim_{\phi_{2}}=\left\{\left\{x_{1}, x_{2}\right\},\left\{x_{3}, x_{4}\right\},\left\{x_{5}, x_{6}\right\}\right\}, O / \sim_{\left\{\phi_{1}, \phi_{2}\right\}}=\left\{\left\{x_{1}, x_{2}\right\},\left\{x_{3}\right\},\left\{x_{4}\right\},\left\{x_{5}, x_{6}\right\}\right\}$. Let sets $X, Y \subseteq O, B_{1}, B_{2} \subseteq F$ be defined as: $X=\left\{x_{2}, x_{3}, x_{4}\right\}, Y=\left\{x_{3}, x_{4}, x_{6}\right\} B_{1}=$ $\left\{\phi_{2}\right\}, B_{2}=\left\{\phi_{1}, \phi_{2}\right\}$. Since $\left\{x_{3}, x_{4}\right\} \in O / \sim_{\left\{\phi_{2}\right\}}$ and $\left\{x_{3}, x_{4}\right\} \subseteq X, Y$ then $X \bowtie_{\sim_{B_{1}}} Y$. Since $\left\{x_{4}\right\} \in O / \sim_{\left\{\phi_{1}, \phi_{2}\right\}}$ and $\left\{x_{4}\right\} \subseteq\left\{x_{3}, x_{4}\right\} \subseteq X, Y$ then $X \bowtie_{\sim_{B_{2}}} Y$.

4.13. Definition. Let $\langle O, F\rangle$ be a perceptual system and $F=B_{n}=\left\{\phi_{1}, \phi_{2}, \ldots, \phi_{n}\right\}$.

$$
B_{1} \subseteq B_{2} \subseteq \ldots \subseteq B_{n}
$$

Then the ascending subsets (4.1) is called as a chain of probe function sets or briefly a feature sets chain.

From Proposition 4.6, we can give following proposition.

4.14. Proposition. Let $\langle O, F\rangle$ be a perceptual system , $F=B_{n}=\left\{\phi_{1}, \phi_{2}, \ldots, \phi_{n}\right\}$ and $B_{1} \subseteq B_{2} \subseteq \ldots \subseteq B_{n}$ be a feature chain. Then the followings hold: 
(1) $\prod\left(O, \sim_{B_{1}}\right) \subseteq \prod\left(O, \sim_{B_{2}}\right) \subseteq \ldots \subseteq \prod\left(O, \sim_{F}\right)$

(2) $\prod\left(O, B_{1}\right) \subseteq \prod\left(O, B_{2}\right) \subseteq \ldots \subseteq \prod(O, F)$.

4.15. Definition. Let $\langle O, F\rangle$ be a perceptual system and $F=B_{n}=\left\{\phi_{1}, \phi_{2}, \ldots, \phi_{n}\right\}$.

$$
\bowtie_{B_{1}} \subseteq \bowtie_{B_{2}} \subseteq \ldots \subseteq \bowtie_{F}
$$

The relation (4.2) corresponding to (4.1) is called as chain of a perceptual nearness or briefly nearness chain.

From Proposition 4.7 and Proposition 4.9 we can give following proposition.

4.16. Proposition. Let $\langle O, F\rangle$ be a perceptual system $, F=B_{n}=\left\{\phi_{1}, \phi_{2}, \ldots, \phi_{n}\right\}, X, Y \subseteq$ $O$ and $\aleph_{B_{1}} \subseteq \aleph_{B_{2}} \subseteq \ldots \subseteq \aleph_{F}$ a nearness chain. Then the following conditions hold:

(1) $X \bowtie_{B_{1}} Y \Rightarrow X \bowtie_{B_{2}} Y \Rightarrow \ldots \Rightarrow X \bowtie_{F} Y$

(2) $\operatorname{Near}_{B_{1}}(O) \subseteq \operatorname{Near}_{B_{2}}(O) \subseteq \ldots \subseteq N e a r_{F}(O)$.

4.17. Definition. Let $\langle O, F\rangle$ be a perceptual system and $F=B_{n}=\left\{\phi_{1}, \phi_{2}, \ldots, \phi_{n}\right\}$.

$$
\sim_{F} \subseteq \sim_{B_{n-1}} \subseteq \ldots \subseteq \sim_{B_{1}}
$$

The relation (4.3) corresponding to (4.1) is called a chain of indiscernibility relations or briefly indiscernibility chain.

4.18. Remark. By using Definition 4.15 and Definition 4.17 , we obtain $\aleph_{\sim_{B_{1}}} \subseteq \bowtie_{\sim_{B_{2}}} \subseteq$ $\ldots \subseteq \bowtie_{\sim_{F}}$. In fact, more than one indiscernibility chain can be formed. We can imagine this indiscernibility chain as a tree, i.e., a branching model which is formed by trunk, branch, thinner branch and so on, respectively. So we get a tree which has $n$ features in the trunk and 1 feature in the thinnest branch.

From Proposition 4.11 we can give following proposition.

4.19. Proposition. Let $\langle O, F\rangle$ be a perceptual system, $X, Y \subseteq O, F=B_{n}=\left\{\phi_{1}, \phi_{2}, \ldots, \phi_{n}\right\}$

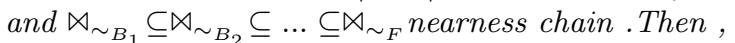

$X \bowtie_{\sim_{B_{1}}} Y \subseteq X \bowtie_{\sim_{B_{2}}} Y \subseteq \ldots \subseteq X \bowtie_{\sim_{F}} Y$.

4.20. Remark. There is a nuance between $X \bowtie_{F} Y$ and $X \bowtie_{\sim_{F}} Y . X \bowtie_{\sim_{F}} Y$ implies that the sets $X$ and $Y$ near to each other with respect to only the $\sim_{F}$ indiscernebility relation in $\langle O, F\rangle$ perceptual system. However, $X \bowtie_{F} Y$ implies that the sets $X$ and $Y$ near to each other by means of Definition 2.10 .

\section{References}

[1] Henry,C. Neighbourhoods, Classes and Near Sets, Applied Mathematical Sciences, Vol. 5, no. $35,1727-1732,2011$.

[2] Henry,C. Near Sets: Theory and Aplications, (Ph.D. Diss., supervisors: J. F. Peters, Dept. Elec. and Comp. Engg., U. of Manitoba, WPG, MB, Canada, 2010).

[3] İnan, E., Öztürk, M., Near groups on nearness approximation spaces, Hacettepe Journal of Mathematics and Statistics 4, 545-558, 2012.

[4] Öztürk, M.,İnan, E., Soft nearness approximation spaces, Fund. Informaticae (2014) to appear.

[5] Pawlak, Z. and Skowron, A. Rudiments of rough sets, Inform. Sci. 177, 3-27, 2007.

[6] Pawlak, Z. Rough sets, Int. J. Comput. Inform. Sci. 11, 341-356, 1981. 
[7] Pawlak, Z. Rough sets - theoretical aspects of reasoning about data, (Kluwer Academic Publishers, Dordrecht, 1991).

[8] Pawlak, Z. Classification of objects by means of attributes, Polish Acad. Sci., 429, 1981.

[9] Peters, J. F. and Wasilewsk,P. Foundations of near sets, Information Sciences 179 , 30913109, 2009.

[10] Peters, J. F. Classification of objects by means of features, (in: Proceedings of the IEEE Symposium Series on Foundations of Computational Intelligence (IEEE SCCI 2007), Honolulu, Hawaii, 2007).

[11] Peters, J. F. Near sets, General theory about nearness of objects, Appl. Math. Sci. 1 (53), 2029-2609, 2007.

[12] Peters, J. F. and Pal, S. K. Cantor, Fuzzy, Near, and Rough Sets in Image Analysis, (In: Pal, S.K. and Peters, J. F.(eds.) Rough Fuzzy Image Analysis: Foundations and Methodologies, CRC Pres ,Taylor and Francis Group, Boca Raton, U.S.A, 2010), 1.1-1.16.

[13] Peters, J.F. Near sets, special theory about nearness of objects, Fundam. Inform. 75 (1-4), 407-433, 2007.

[14] Peters, J. F. Classification of perceptual objects by means of features, Int. J. Info. Technol. Intell. Comput. 3 (2), 1-35, 2008.

[15] Peters, J. F. Fuzzy Sets, Near Sets, and Rough Sets for Your Computational Intelligence Toolbox, Foundations of Comput. Intel. Vol. 2, SCI 202, pp. 3-25, 2009.

[16] Peters, J. F. and Ramanna, R. Feature selection: a near set approach, (in: ECML \& PKDD Workshop on Mining Complex Data, Warsaw, 2007), pp. 1-12.

[17] Peters, J. F., Topology of Digital Images. Visual Pattern Discovery in Proximity Spaces, Intelligent Systems Reference Library, Springer, ISBN 978-3-642-53844-5, vol. 63, pp. 1-342, 2014.

[18] Peters, J. F., İnan, E., Öztürk, M., Spatial and descriptive isometries in proximity spaces, General Mathematics Notes 21 (2), 125-134, 2014.

[19] Peters, J. F., Naimpally, S., Applications of near sets, Notices of the Amer. Math. Soc. 59 (4), 536-542, DOI: http://dx.doi.org/10.1090/noti817, 2012.

[20] Taşbozan, H., İçen, İ., Bağırmaz, N., Özcan, A., Soft sets and soft topology on nearness approximation spaces, Fund. Informaticae (2014) to appear.

[21] Wolski, W. Perception and classification. A note on near sets and rough sets, Foundamenta Informaticae, 101 , 143-155, 2010. 